\title{
DIRECT MEASUREMENTS OF ANCHOR DAMPING IN PRESSURE-LIMITED RING RESONATORS
}

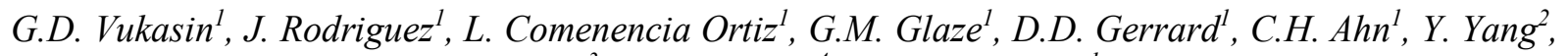 \\ J. Lake ${ }^{3}$, R.N. Candler ${ }^{4}$, and T.W. Kenny ${ }^{1}$ \\ ${ }^{1}$ Stanford University, Stanford, CA, USA, ${ }^{2}$ Integrated Device Technology Incorporated, San Jose, CA, USA, \\ ${ }^{3}$ uBeam, Los Angeles, CA, USA, ${ }^{4}$ University of California, Los Angeles, CA, USA
}

\begin{abstract}
We measure the quality factor (Q) due to gas damping, thermoelastic dissipation (TED), and anchor damping of a ring resonator operating in the wineglass mode. We analyze the Q over a range of temperatures and pressures and compare anchor damping of two different anchor designs. This method provides a path towards better understanding of anchor damping in MEMS.
\end{abstract}

\section{INTRODUCTION}

MEMS resonators lose energy due to gas damping, thermoelastic damping, anchor damping, and the Akhiezer effect. The effect of these damping mechanisms on the performance of MEMS resonators is expressed by the quality factor (Q), which determines fundamental noise in MEMS devices $[1,2]$. The total $\mathrm{Q}$ can be calculated as the reciprocal sum of each damping mechanism:

$\frac{1}{Q}=\sum \frac{1}{Q_{\text {factors }}}=\frac{1}{Q_{\text {Gas }}}+\frac{1}{Q_{T E D}}+\frac{1}{Q_{\text {Anchor }}}+\frac{1}{Q_{\text {Akhiezer }}}+\frac{1}{Q_{\text {others }}}$

Previously, we have studied resonators whose primary damping mechanisms were thermoelastic dissipation (TED) and anchor damping [3]. In this paper, we have found a device that is dominated by gas damping and includes contributions from TED and anchor damping, and we quantitatively identify the contributions from each of these mechanisms.

\section{METHODS}

The device is a ring resonator operated in the $n=2$ wineglass mode at $145 \mathrm{kHz}$, as illustrated in Figure 1. There are two anchor designs for this device. The first is a pure silicon anchor fixed to the center (10 $\mu \mathrm{m}$ in diameter) of the device, which is attached to the epitaxial polysilicon cap. The polysilicon cap is attached to the substrate via the device layer and a layer of silicon oxide. The other design includes anchors at two points on the perimeter ( $3 \mu \mathrm{m}$ wide) of the ring resonator in the device layer, which are connected to the substrate via silicon oxide. Examples of the two anchor designs are shown in Figures 1a and 1b. The die was fabricated with the established epi-seal encapsulation process [4].

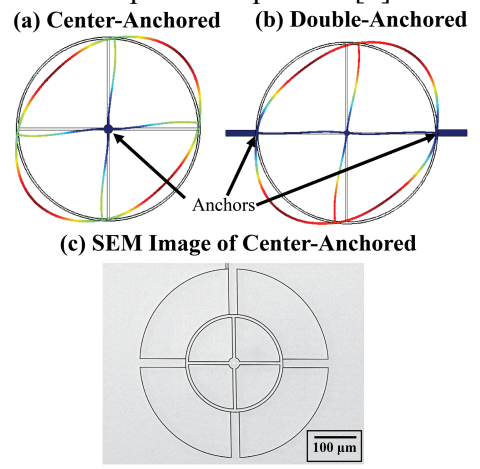

Figure 1: (a) COMSOL simulation of displacement in the wineglass mode $(n=2)$ of center-anchored ring, (b) COMSOL simulation of wineglass mode $(n=2)$ of double-anchored ring. These mode shapes are similar, but not exactly identical. (c) SEM image of centeranchored ring prior to completion of encapsulation process.

For each device, we used a liquid nitrogen cooled ultra-high vacuum (UHV) pressure chamber to study $Q$ as a function of temperature $(85 \mathrm{~K}$ to $300 \mathrm{~K})$ and pressure $\left(10^{-4} \mathrm{~Pa}\right.$ to $\left.10^{-1} \mathrm{~Pa}\right)$. We begin with measurements of $\mathrm{Q}(\mathrm{T})$ for sealed devices, followed by using a focused ion beam (FIB) to vent these sealed devices enabling $\mathrm{Q}(\mathrm{P})$ measurements at room temperature to identify the contribution of pressure damping in the sealed devices. Finally, we measure $\mathrm{Q}(\mathrm{T})$ for vented devices at UHV pressures over temperature.

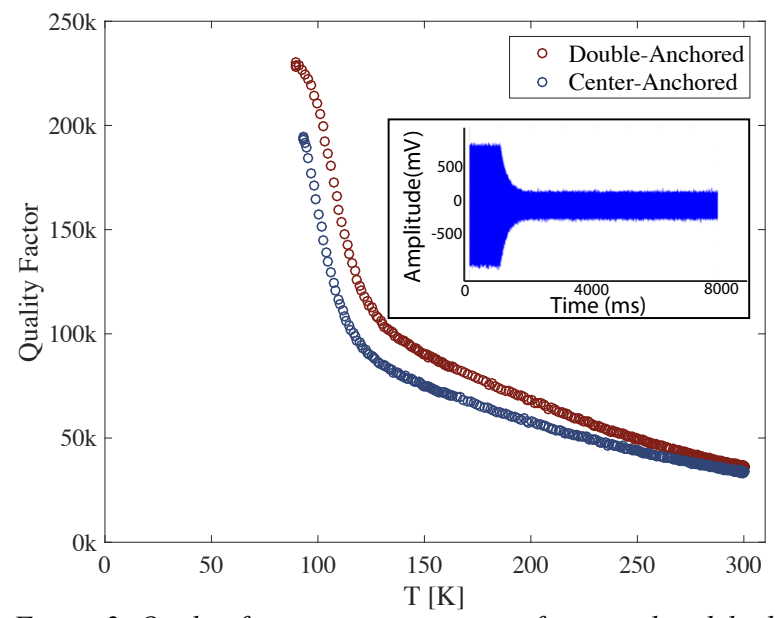

Figure 2: Quality factor vs. temperature of encapsulated double and center-anchored rings. The inset shows a representative ringdown measurement, which is used to calculate $Q$.

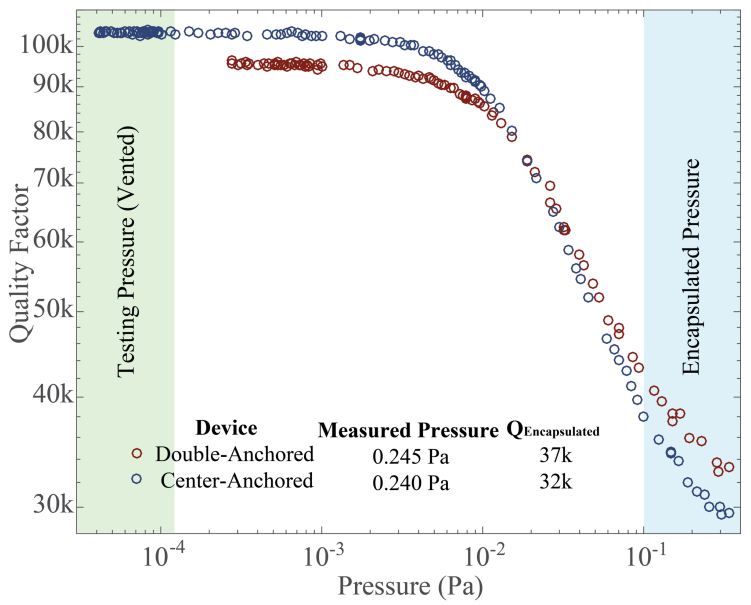

Figure 3: Pressure sweep of double and center-anchored devices at room temperature. The areas indicate the typical pressure range for our encapsulated device (blue) and the pressure where the vented devices were tested (green). 
As described in previous work $[3,5]$ our measurements of $Q(T)$ at low temperature on vented devices reveal $Q$ in the absence of pressure damping (UHV conditions) and TED (the thermal expansion coefficient in $\mathrm{Si}$ crosses zero at $120 \mathrm{~K}$, which disables coupling between mechanical and thermal effects, and therefore disables TED) [6]. Therefore, we directly detect anchor damping at the peak of the $\mathrm{Q}(\mathrm{T})$ curve.

\section{RESULTS}

As seen in Figure 2, Q increases at low temperature for both devices without a $\mathrm{Q}(\mathrm{T})$ peak at $120 \mathrm{~K}$, which would be the unique signature of a strong TED contribution [5]. For both devices, we see a dramatic change in the slope around $140 \mathrm{~K}$, possibly indicating presence of a two-component gas mixture inside this encapsulation.

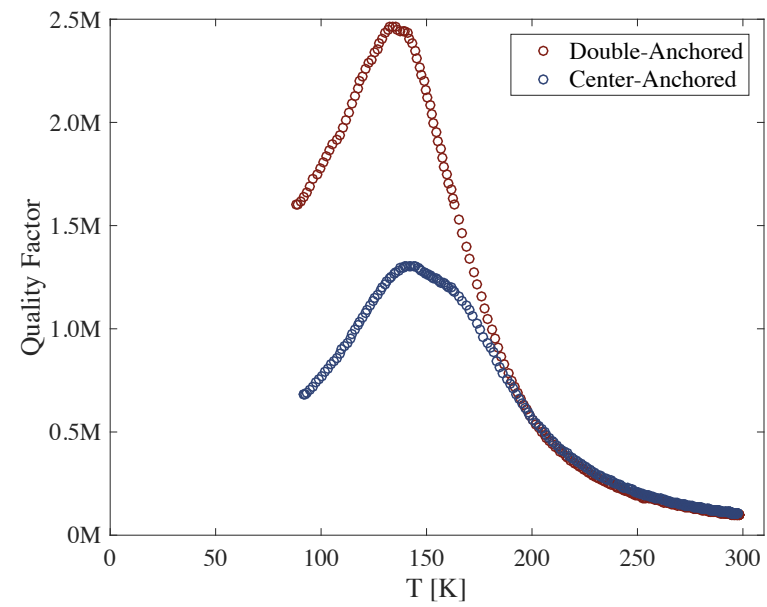

Figure 4: Quality factor vs. temperature of vented double and center-anchored rings. The peak $Q$ of the double-anchored ring is $2.5 \mathrm{M}$ and peak $Q$ of the center-anchored ring is $1.3 \mathrm{M}$. These peaks are a consequence of the zero-crossing in the temperature dependence of the thermal expansion coefficient of Si, which disables TED, leaving only a contribution from anchor damping.

After FIB-venting, Q(P) measurements (Figure 3) indicate that the sealed devices were dominated by pressure damping, and that vented devices operated in vacuum reach a limit from other damping mechanisms. $\mathrm{Q}(\mathrm{T})$ experiments on vented devices of both designs under UHV conditions are shown in Figure 4. These Q(T) measurements for both devices have a profile that is characteristic of TED limited devices [5], with the $\mathrm{Q}$ at the peak in $\mathrm{Q}(\mathrm{T})$ indicating the contribution of anchor damping.

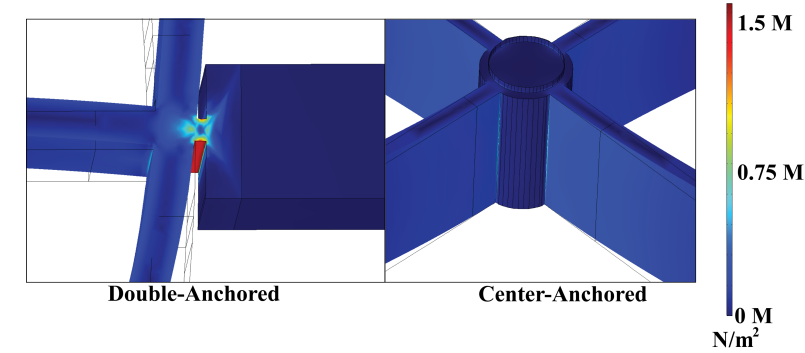

Figure 5: Plots of von Mises stress in the double-anchored device (left) and center-anchored device (right).

These experiments lead to the initially surprising conclusion that the anchor damping of a center-anchored ring resonator is stronger than for a ring resonator with a pair of side anchors. Figure
5 shows simulations of the von Mises stress for both types of anchors. Looking closely at these stress distributions, it is clear that there is significantly more stress trapped in the flexures that connect the rings to the side anchors of the double-anchored device than in the center anchor. The stresses that are not trapped in the anchors must propagate further into the substrate, and would be expected to cause stronger energy loss from the vibrational mode.

Another interesting result is that at UHV, the room-temperature $\mathrm{Q}$ of the center-anchored device is larger (Figure 3). We believe this means that the center-anchored device has more pressure damping than the double-anchored device due to the difference in mode shape of the two devices.

An important result of this study is the recognition that this comprehensive experimental investigation $(\mathrm{Q}(\mathrm{T})$ for sealed devices, $\mathrm{Q}(\mathrm{P})$ for vented devices, $\mathrm{Q}(\mathrm{T})$ for vented devices) was necessary to construct an accurate understanding of the damping mechanisms in these devices. If we had relied only on room temperature measurements, we could not have accurately accounted for TED, and therefore it would have been impossible to make convincing arguments about the anchor damping contribution.

We recommend that a process like this be carried out in any study in which researchers are interested in achieving a complete and accurate understanding of damping mechanisms in their devices.

\section{CONCLUSION}

Measuring $\mathrm{Q}(\mathrm{T})$ over a wide temperature range is the best path to reliable identification and quantification of damping mechanisms in MEMS resonators. For the ring resonators studied here, we demonstrate this method and provide the first direct measurement of anchor damping in these devices. At the conclusion of this work, we were surprised to find that the single, center-anchored resonator had stronger anchor damping than the dual-side anchored design. Looking ahead, we will use experiments like this on many more resonators to build capability for quantitative prediction of anchor damping in MEMS.

\section{REFERENCES}

[1] J. T. M. van Beek and R. Puers, "A review of MEMS oscillators for frequency reference and timing applications", Journal of Micromechanics and Microengineering, 22, 1 (2012).

[2] T. B. Gabrielson, "Mechanical-thermal noise in micromachined acoustic and vibration sensors", IEEE Transactions on Electron Devices, 40, 5 (1993).

[3] J. Rodriguez, D.D. Gerrard, G.M. Glaze, S. Chandorkar, L. Comenencia, Y. Chen, I. B. Flader, T.W. Kenny, "Direct measurements of anchor damping in MEMS resonators", Technical Digest of the 2017 IEEE Sensors, Glasgow, Scotland, UK, 10/30 - 11/1/17, IEEE (2017), pp. 1-3.

[4] E A. Partridge and M. Lutz, "Epi-seal pressure sensor and method for making an episeal pressure sensor", U.S. Patent 6928879, 2005., U.S. Patent 6928879, 2005.

[5] J. Rodriguez, D. D. Gerrard, S. Chandorkar, Y. Chen, G. M. Glaze, I. B. Flader, C. H. Ahn, E. J. Ng, and T. W. Kenny, "Wide-range temperature dependence studies for devices limited by thermoelastic dissipation and anchor damping", Technical Digest of the 2017 19th International Conference on Solid-State Sensors, Actuators and Microsystems, Kaohsiung, Taiwan, 6/18-22/17, IEEE and Electron Devices Society (2017), pp. 1100-1103.

[6] T. Middelmann, A. Walkov, G. Bartl, and R. Schödel, "Thermal expansion coefficient of single-crystal silicon from 7 K to 293 K”, Phys. Rev. B, 92, 17 (2015). 